\title{
Fostering hope and resilience amidst intractable ethical dilemmas brought about by the COVID-19 pandemic
}

\author{
Allen Alvarez, Espen Dyrness Stabell, Gitte Koksvik, May Thorseth
}

Prolonged and unprecedented implementation of public health protection measures has created intractable value conflicts in all societies around the world. Governments are faced with tough decisions, pitting economic stability against protecting public health through broad restrictions and extended lockdowns. In a short period of time, new resources were created, including huge public spending to mitigate the impact of the pandemic on people's livelihood. The dramatic rise in critically ill patients pushed healthcare systems to increase the number of ICU beds beyond the capacity of healthcare workers (Robert et al. 2020). The conflict between duty to provide care according to professional standards and attending to huge numbers of patients disproportional to staffing capacity has highlighted the intractable dilemma between ethics principles that pushed decision-makers to face the reality that a single satisfactory solution does not exist (Robert et al. 2020). Each of the competing options can be defended with strong reasons as well as criticized for their weak points, especially the apparent unacceptable outcomes to which they may lead. Adopting less than perfect solutions because there are no perfect options causes moral distress not only to decision-makers but to all those involved in implementing those decisions, including healthcare workers who witness how these critical decisions can impact the psychological well-being of patients, relatives and caregivers. These undesirable outcomes are beyond the conventional scope of wellbeing that healthcare aims to affect. They also seem to go beyond the scope of our ethical reflection and analysis as they are deemed acceptable trade-offs in decisions made in extreme scarcity.

In view of how the tough decisions made in society can impact the well-being of healthcare workers-whom we need to help us survive the pandemic-it is crucial that we include their voices and perspectives (Morley et al. 2020). Every opportunity to include them in conversations that inform high-stakes decisions about operational changes in healthcare is ethically essential not only for epistemic reasons, i.e. to gather relevant facts from a point of care perspective, but also for reasons relevant to their well-being, especially their mental health. Policy makers, leaders of healthcare organizations and ministries of health should make it a requirement that all operational changes impacting healthcare workers should be informed by robust stakeholder engagement. This will enable healthcare workers and their representatives to increase awareness of the intractable value conflicts that the decisions involve, as well as voice their unique perspectives and challenges to 
decision-makers. Facilitated focus group discussions among healthcare workers enable ethical analysis and reflection to draw upon the values of those who experience these ethical challenges at the bedside (Verkerk and Lindemann 2012). These forms of stakeholder engagement also widen the scope of considerations to include relationships that are affected, as well as the emotional impact of losses and gains implied by decisions. Although we may not be able to make perfect decisions, including the perspectives of all relevant stakeholders in these conversations can help distribute the weight of the losses we may incur and help increase the legitimacy of these tough decisions by including those who will be involved implementing them.

Hope does not mean the absence of grief and loss. Rather, it is the acknowledgment that beyond these losses, we are making careful and collaborative decisions to carry the burden of unavoidable grief that arises from trade-offs in the face of ethical dilemma. This collaborative approach to hope also fosters the type of solidarity we need to survive the pandemic. It makes us more aware of how the pandemic is affecting all of us in specific ways that triggers compassionate response. This can help create opportunities for reciprocity; if we feel heard we are more likely to be more compassionate to others as well. Ethical decision-making that is inclusive of stakeholders can help in employing "a proactive approach in mitigating the lasting effects of moral distress" by creating and promoting safety, calmness, connectedness, self-efficacy and hope (Williams, Brundage, Williams 2020: 65). This awareness can also help motivate peer support and the use of psychological first aid to help patients, colleagues and even themselves in the face of crisis. All sectors of society, including healthcare, have been stretched to the limit. Applied ethics may need to do its share of going beyond our conventional scope practice of ethical reflection and analysis to attend to pandemic needs.

This special issue of Etikk i Praksis - Nordic Journal of Applied Ethics features four articles that address a number of urgent ethical issues that arise in the COVID19 pandemic.

The paper by Sindre Horn, Mathias Barra, Ole Frithjof Norheim, and Carl Tollef Solberg titled Public health priority setting: A case for priority to the worse off in well-being during the COVID-19 pandemic evaluates potential priority-setting criteria for public health interventions. The authors argue that we should follow three criteria for public health priority setting: benefit, resources and improving the well-being of the worse off-instead of the three official criteria of health benefit, resources and severity followed in Norway. They argue that benefits and priority to the worse off may reasonably be understood in terms of individual well-being, rather than only health, for public health priority setting.

Elias Moser, in the second article Against Draconian Penalties for COVID-19 Quarantine Infringements, argues that severe penalties imposed upon those who violate quarantine measures are extremely unjust. Moser argues that governmental institutions should intensify controls instead by increasing surveillance of compliance with self-isolation obligations. While the author acknowledges that surveillance may be labor-intensive and costly, it is a way to fulfill the government's obligation to its citizens. It may even be a more effective way to prevent or decrease the further spread of COVID-19 in the population since it will push people to comply with quarantine measures, knowing they will be watched closely. 
In The "foreign" virus? Justifying Norway's Border Closure, Attila Tanyi and Magnus Skytterholm Egan discuss Norway's border policy during the pandemic. Facing the threat of "imported infection," the Norwegian government has introduced the strictest rules on entering the country since World War II. The rules introduced in January 2021 - which were the strictest since the pandemic broke out in Norway in March 2020-denied entry for most foreign citizens. The authors look at consequences of the rules for groups, such as transnational citizens, international migrants who have attachments and connections in other countries and Norwegian residents who have attachments abroad, and critically examine the justification given for the rules. They argue that while many border restrictions are legitimate, the recent banning of entry has not been adequately justified. Furthermore, they argue, the ban unduly limits personal freedoms and places an unjust burden on transnational citizens and Norwegians with close relations abroad.

Nathan Emmerich and Pat McConville in Reverse Triage and People Whose Disabilities Render Them Dependent on Ventilators: Phenomenology, Embodiment and Homelikeness, address a specific concern that has arisen in relation to proposals to potentially reallocate ventilators relied upon by disabled or chronically ill patients. This issue is examined via three morally parallel scenarios. The paper suggests that whilst property rights cannot resolve these scenarios in a satisfactory manner, it may be possible to do so if we draw on the resources of phenomenology. In contradistinction to a recent paper on this topic (Reynolds et al. 2021), the authors argue that ethical claims to ventilators are not well grounded by the notion that they are embodied objects. Instead, they suggest the alternative phenomenological notion of homelikeness, arguing that the personal ventilators of individuals who commonly rely upon them become part of their ordinary, everyday or homelike being. They are a necessary part of the continuation or maintenance of their basic state of health or wellbeing, and the reallocation of such objects is unethical.

This issue of Etikk $i$ praksis also includes a commentary to a previously published article. Krister Bykvist presents a response and appraisal of the arguments in the article Two asymmetries in population and general normative ethics by Mat Rozas.

We thank the reviewers and contributors to this publication as well as all of those who participated in the summer workshop that inspired the development of this special issue. It is our hope that the papers included here will contribute to resilient thinking to help address the ongoing ethical challenges we face amidst the COVID-19 pandemic.

\section{References}

Morley, G., Grady, C., McCarthy, J., \& Ulrich, C. M. (2020). COVID-19: ethical challenges for nurses. Hastings Center Report, 50(3), 35-39. https://doi.org/ 10.1002/hast.1110

Robert, R., Kentish-Barnes, N., Boyer, A., Laurent, A., Azoulay, E., \& Reignier, J. (2020). Ethical dilemmas due to the COVID-19 pandemic. Annals of intensive care, 10(1), 1-9. https://doi.org/10.1186/s13613-020-00702-7 
Williams, R. D., Brundage, J. A., \& Williams, E. B. (2020). Moral injury in times of COVID-19. Journal of health service psychology, 46(2), 65-69. https://doi.org/ 10.1007/s42843-020-00011-4

Verkerk, M., \& Lindemann, H. (2012). Toward a Naturalized Clinical Ethics. Kennedy Institute of Ethics Journal 22(4), 289306. https://www.muse.jhu.edu/article/495156 